\title{
SSP IMEX Runge-Kutta WENO Scheme for Generalized Rosenau-KdV-RLW Equation
}

\author{
Muyassar Ahmat and Jianxian Qiu* \\ School of Mathematical Sciences and Fujian Provincial Key Laboratory of \\ Mathematical Modeling and High-Performance Scientific Computing, \\ Xiamen University, Xiamen 361005, China.
}

Received June 8, 2020; Accepted November 4, 2020;

Published online January 21, 2022.

\begin{abstract}
In this article, we present a third-order weighted essentially non-oscillatory (WENO) method for generalized Rosenau-KdV-RLW equation. The third order finite difference WENO reconstruction and central finite differences are applied to discrete advection terms and other terms, respectively, in spatial discretization. In order to achieve the third order accuracy both in space and time, four stage third-order L-stable SSP Implicit-Explicit Runge-Kutta method (Third-order SSP EXRK method and thirdorder DIRK method) is applied to temporal discretization. The high order accuracy and essentially non-oscillatory property of finite difference WENO reconstruction are shown for solitary wave and shock wave for Rosenau-KdV and Rosenau-KdV-RLW equations. The efficiency, reliability and excellent SSP property of the numerical scheme are demonstrated by several numerical experiments with large CFL number.
\end{abstract}

AMS subject classifications: 65M60, 35L65

Key words: Rosenau-KdV-RLW equation, WENO reconstruction, finite difference method, SSP implicit-explicit Runge-Kutta method.

\section{Introduction}

The nonlinear wave behavior is one of the active scientific research areas during the past several decades. Numerical solution of nonlinear wave equations is significantly necessary since most of these types of equations are not solvable analytically in the case of the nonlinear terms are included.

Many mathematical models, especially nonlinear partial differential equations describe various types of wave behavior in nature. Typically, the KdV equation (Kortewegde Vries equation) is suitable for small-amplitude long waves on the surface of the subject, such as shallow water waves, ion sound waves, and longitudinal astigmatic waves.

*Corresponding author. Email addresses: muyassar@stu.xmu.edu.cn (Ahmat M), jxqiu@xmu.edu.cn (Qiu J) 
RLW equation (Regularized Long-Wave equation) can describe not only shallow water waves, but also nonlinear dispersive waves, ion-acoustic plasma waves, magnetohydrodynamic plasma waves. The Rosenau equation [1] was proposed for explaining the dynamic of dense discrete systems since the case of wave-wave and wave-wall interactions can not be explained by the KdV and RLW equations.

In order to further consider the nonlinear wave behavior, the viscous term $u_{x x x}$ or $u_{x x t}$ need to be included in Rosenau equation, which leads to the achievement of RosenauRLW equation:

$$
u_{t}+\alpha u_{x}+\delta u_{x x t}+v u_{x x x x t}+\varepsilon\left(u^{p}\right)_{x}=0 .
$$

or Rosenau-KdV equation:

$$
u_{t}+v u_{x x x x t}+\alpha u_{x}+\theta u_{x x x}+\varepsilon\left(u^{p}\right)_{x}=0 .
$$

There have been many difficulties in evaluating analytical solutions of nonlinear dispersive wave equations and so on the development of numerical schemes. Even so, one derived the solitary wave solution and singular soliton solution for the Rosenau-KdV equation by the ansatz method as well as the semi-inverse variational principle [2] while the shock wave solution of this equation was determined for two particular values of the power law nonlinearity parameter $p=3$ and $p=5$ by Ebadi [3].

Significant numerical studies have been done on the Rosenau-KdV equation [4,5]. Two-level nonlinear implicit Crank-Nicolson difference scheme and three-level linearimplicit difference scheme were presented to solve two-dimensional generalized Rosenau$\mathrm{KdV}$ equation by Atouani [4]. Their experiment proved that both schemes were uniquely solvable, unconditionally stable and second-order convergent in $L_{1}$ norm, the linearized scheme was more effective in terms of accuracy and computational cost. Wang and Dai [5] proposed a conservative unconditionally stable finite difference scheme with $\mathrm{O}\left(h^{4}+\tau^{2}\right)$ for the generalized Rosenau-KdV equation in both one and two dimension, where $h$ is spatial step and $\tau$ is temporal step, respectively.

A mass-preserving scheme which combined a high-order compact scheme and a threelevel average difference iterative algorithm was analyzed and tested for the RosenauRLW equation in [6]. In their work, they focused on the development of the approach for solving the nonlinear implicit scheme in aim to improve the accuracy of approximate solutions. The Rosenau-RLW equation was also solved by second-order nonlinear finite element Galerkin-Crank-Nicolson method which was linearized by predictor-correction extrapolation technique in [7]. An energy conservative two-level fourth-order nonlinear implicit compact difference scheme for three dimensional Rosenau-RLW equation was designed by $\mathrm{Li}$ [8] and an iterative algorithm was introduced to generate this nonlinear algebraical system.

In this paper, we focus on one-dimensional generalized Rosenau-KdV-RLW equation. This model is difficult to solve numerically because of the excessive computational cost caused by high order mixed derivative term and the selective wave behavior caused by the power law nonlinearity term. In order to keep this model in a generalized setting, the 
Rosenau-KdV-RLW equation is written as:

$$
u_{t}+\delta u_{x x t}+v u_{x x x x t}+\alpha u_{x}+\theta u_{x x x}+\varepsilon\left(u^{p}\right)_{x}=0 .
$$

where $u(x, t)$ denote the profile of the wave while $x$ and $t$ are the spatial and temporal variables, respectively. $\alpha>0, \varepsilon>0$ are the parameters of linear and nonlinear advection terms, $p \geq 2$ is the parameter of power law nonlinearity. $\theta, \delta, v$ are the parameters of $\mathrm{KdV}$, RLW, Rosenau terms, respectively.

Rosenau-KdV-RLW equation has been studied both theoretically and numerically in recent years. Ansatz approach and semi-inverse variational principle were used to determine the solitary and shock solution, and the conservation laws of the Rosenau-KdVRLW equation with power law nonlinearity were computed by the aid of multiplier approach in Lie symmetry analysis in [9] and [10]. A three-level second-order accurate weighted average implicit finite difference scheme was presented by Wongsaijai [11] to solve the Rosenau-KdV-RLW equation. Wang [12] introduced a three-level linear conservative implicit finite difference scheme for solving this equation which was easy to implement and had simple computational structure. A multi-symplectic scheme and an energy-preserving scheme based on the multi-symplectic Hamiltonian formulation of the equation were tested for the generalized Rosenau-type equation in [13]. These methods were implemented efficiently by the discrete fast Fourier transform with spectral accuracy in space while second-order accuracy in time.

To the best of our knowledge, many numerical schemes are employed to simulate the solitary wave of the Rosenau-KdV and Rosenau-KdV-RLW equations. But as far as we know, there is very few numerical scheme has bees presented for the shock wave of these equations. In this paper, We're going to fill this gap effectively.

The Implicit-Explicit (IMEX) Runge-Kutta method is an effective time solver with the advantages of loosening the CFL restriction caused by the Explicit scheme and reducing the computational cost caused by Implicit method reasonably for PDEs which contains stiff and non-stiff terms all together, and applied generally for this type of PDEs [15-17]. In order to ensure the stability stands for this type of large ODE system obtained from spatial discretization, It is much safer to use IMEX Runge-Kutta methods with strong stability preserving (SSP) properties [18-20].

The weighted essentially non-oscillatory (WENO) method is mostly applied for hyperbolic conservation laws with the advantages of the capability to achieve high-order accuracy in smooth regions while maintaining stable, non-oscillatory property in sharp or stiff region [21-23]. Here, we use the same approach for the solitary wave solution and shock wave solution of Rosenau-KdV-RLW equation.

The advantages of finite difference WENO reconstruction [22] is exploited in wave motions, especially shock wave for Rosenau-KdV equation and Rosenau-KdV-RLW equation with power law nonlinearity parameter $p=3$ and $p=5$ as given in $[3,9]$ to deal with stiff wave motion. Instead of using third order TVD Runge-Kutta scheme in time direction, we choose the SSP IMEX Runge-Kutta scheme [20] to avoid the strict CFL restriction and large computational cost. To be specific, we use third-order finite 
difference WENO scheme for advection terms of (1.3) and treat explicitly in the time direction. The rest of (1.3) is treated by high order central finite difference method in space and treated implicitly in time.

The paper is arranged as follows. In Section 2, the third-order finite difference WENO scheme and high order finite difference method are performed. In Section 3, the thirdorder SSP IMEX Runge-Kutta scheme is given for the treatment in time. Extensive numerical results are proposed in Section 4 to illustrate the accuracy and efficiency of the present method. Concluding remarks are given in the final section.

\section{Spatial discretization}

We use a uniform mesh of cell size $h$ in space. The uniform mesh is distributed as follows:

$$
\begin{aligned}
& x \in\left[x_{l}, x_{r}\right], \quad x_{i}=x_{l}+i h, \quad i=1: N-1, \quad x_{1}=x_{l}, \quad x_{N}=x_{r}, \\
& I_{i}=\left[x_{i-\frac{1}{2}}, x_{i+\frac{1}{2}}\right], \quad x_{i+\frac{1}{2}}=\frac{x_{i+1}+x_{i-1}}{2} .
\end{aligned}
$$

We will give a brief sketch of the algorithms about third order finite difference WENO scheme with Lax-Friedrichs flux splitting which is used to treat $f(u)=a u+\varepsilon u^{p}$, here $f(u)_{x}$ can be reformulated as

$$
\left.f(u)_{x}\right|_{x=x_{i}} \approx \frac{1}{h}\left(\hat{f}_{i+\frac{1}{2}}-\hat{f}_{i-\frac{1}{2}}\right) .
$$

where $\hat{f}_{i+\frac{1}{2}}, \hat{f}_{i-\frac{1}{2}}$ are the numerical fluxes such that right hand side of (2.1) is a third order approximation to $\left.f(u)_{x}\right|_{x=x_{i}} \cdot u_{i}(t)$ is defined as a nodal point value $u\left(x_{i}, t\right)$.

In finite difference WENO reconstruction, flux splitting has to be done for the purpose of stability. For flux $\mathrm{f}(\mathrm{u})$, we perform the "Lax-Friedrichs flux splitting" :

$$
f^{+}(u)=\frac{1}{2}(f(u)+\alpha u), \quad f^{-}(u)=\frac{1}{2}(f(u)-\alpha u),
$$

where $\alpha=\max _{u}\left|f^{\prime}(u)\right|$, so that

$$
f(u)=f^{-}(u)+f^{+}(u),
$$

satisfying

$$
\frac{d}{d u} f^{+}(u) \geq 0, \quad \frac{d}{d u} f^{-}(u) \leq 0 .
$$

In here, we only recall the reconstruction of $f^{+}(u)$ at point $x_{i+\frac{1}{2}}$. We choose big stencil $\Gamma=\left[I_{i-1}, I_{i}, I_{i+1}\right]$. In this stencil, we can obtain a second degree polynomial $H(x)$ which is based on the nodal point information of the flux splitting and satisfying:

$$
\frac{1}{h} \int_{x_{j-\frac{1}{2}}}^{x_{j+\frac{1}{2}}} H(x) d x=f_{j}^{+}, \quad j=i-1, i, i+1 \Rightarrow H\left(x_{i+\frac{1}{2}}\right)=-\frac{1}{6} f_{i-1}^{+}+\frac{5}{6} f_{i}^{+}+\frac{1}{3} f_{i+1}^{+} .
$$


In two small stencil $\Gamma_{1}=\left[I_{i-1}, I_{i}\right], \Gamma_{2}=\left[I_{i}, I_{i+1}\right]$, we can obtain two linear polynomial $H_{1}(x), H_{2}(x)$ respectively.

$$
\begin{aligned}
& \frac{1}{h} \int_{x_{j-\frac{1}{2}}}^{x_{j+\frac{1}{2}}} H_{1}(x) d x=f_{j}^{+}, \quad j=i-1, i \Rightarrow H_{1}\left(x_{i+\frac{1}{2}}\right)=-\frac{1}{2} f_{i-1}^{+}+\frac{3}{2} f_{i}^{+}, \\
& \frac{1}{h} \int_{x_{j-\frac{1}{2}}}^{x_{j+\frac{1}{2}}} H_{2}(x) d x=f_{j}^{+}, \quad j=i, i+1 \Rightarrow H_{2}\left(x_{i+\frac{1}{2}}\right)=\frac{1}{2} f_{i}^{+}+\frac{1}{2} f_{i+1}^{+} .
\end{aligned}
$$

Define the linear weights $r_{1}, r_{2}$, such that

$$
H\left(x_{i+\frac{1}{2}}\right)=r_{1} H_{1}\left(x_{i+\frac{1}{2}}\right)+r_{2} H_{2}\left(x_{i+\frac{1}{2}}\right) .
$$

We have $r_{1}=\frac{1}{3}, r_{2}=\frac{2}{3}$. The smoothness indicators to measure the smoothness of $H_{1}(x)$ and $H_{2}(x)$ are defined as

$$
\beta_{1}=\left(f_{i}^{+}-f_{i-1}^{+}\right)^{2}, \quad \beta_{2}=\left(f_{i+1}^{+}-f_{i}^{+}\right)^{2} .
$$

then we define the nonlinear weights as follows:

$$
w_{j}=\frac{\tilde{w}_{j}}{\tilde{w}_{1}+\tilde{w}_{2}}, \quad \tilde{w}_{j}=\frac{r_{j}}{\left(\epsilon+\beta_{j}\right)^{2}}, \quad j=1,2 .
$$

where $\epsilon$ is very small and positive, which is chosen to avoid the denominator becoming 0 and typically chosen to be $\epsilon=10^{-6}$ in the calculation.

Finally, we obtain the third-order approximation:

$$
\hat{f}_{i+\frac{1}{2}}^{+}=w_{1} H_{1}\left(x_{i+\frac{1}{2}}\right)+w_{2} H_{2}\left(x_{i+\frac{1}{2}}\right) .
$$

$\hat{f}_{i+\frac{1}{2}}^{-}$also can be obtained in the same way, so that

$$
\hat{f}_{i+\frac{1}{2}}=\hat{f}_{i+\frac{1}{2}}^{+}+\hat{f}_{i+\frac{1}{2}}^{-} \text {. }
$$

For the second, third and fourth derivative at $\left(x_{i}, t\right)$ in (1.3), we simply use high order central finite difference to approximate them.

$$
\begin{aligned}
& \frac{-\frac{1}{12} u_{i+2}+\frac{4}{3} u_{i+1}-\frac{5}{2} u_{i}+\frac{4}{3} u_{i-1}-\frac{1}{12} u_{i-2}}{h^{2}}=\left(\frac{\partial^{2} u}{\partial x^{2}}\right)_{i}+O\left(h^{4}\right)_{,} \\
& \frac{-\frac{1}{8} u_{i+3}+u_{i+2}-\frac{13}{8} u_{i+1}+\frac{13}{8} u_{i-1}-u_{i-2}+\frac{1}{8} u_{i-3}}{h^{3}}=\left(\frac{\partial^{3} u}{\partial x^{3}}\right)_{i}+O\left(h^{4}\right)_{,} \\
& \frac{-\frac{1}{6} u_{i+3}+2 u_{i+2}-\frac{13}{2} u_{i+1}+\frac{28}{3} u_{i}-\frac{13}{2} u_{i-1}+2 u_{i-2}-\frac{1}{6} u_{i-3}}{h^{4}}=\left(\frac{\partial^{4} u}{\partial x^{4}}\right)_{i}+O\left(h^{4}\right) .
\end{aligned}
$$




\section{The third order SSP IMEX Runge-Kutta method}

We now rewrite (1.3) as

$$
\left(u+\delta u_{x x}+v u_{x x x x}\right)_{t}=-\alpha u_{x}-\theta u_{x x x}-\varepsilon\left(u^{p}\right)_{x} .
$$

and obtain the semi-discrete form by discretizing $(3.1)$ at $\left(x_{i}, t\right)$ :

$$
\left(u_{i}+\delta\left(u_{x x}\right)_{i}+v\left(u_{x x x x}\right)_{i}\right)_{t}=-\alpha\left(u_{x}\right)_{i}-\varepsilon\left(\left(u^{p}\right)_{x}\right)_{i}-\theta\left(u_{x x x}\right)_{i}
$$

We use the above third-order finite difference WENO reconstruction and finite difference discretization procedure for (3.2):

$$
\begin{aligned}
& \frac{d}{d t}\left[u_{i}+\delta \frac{-\frac{1}{12} u_{i+2}+\frac{4}{3} u_{i+1}-\frac{5}{2} u_{i}+\frac{4}{3} u_{i-1}-\frac{1}{12} u_{i-2}}{h^{2}}\right. \\
& \left.\quad+v \frac{-\frac{1}{6} u_{i+3}+2 u_{i+2}-\frac{13}{2} u_{i+1}+\frac{28}{3} u_{i}-\frac{13}{2} u_{i-1}+2 u_{i-2}-\frac{1}{6} u_{i-3}}{h^{4}}\right] \\
& =L\left(u\left(x_{i}, t\right)\right)+\left[-\theta \frac{-\frac{1}{8} u_{i+3}+u_{i+2}-\frac{13}{8} u_{i+1}+\frac{13}{8} u_{i-1}-u_{i-2}+\frac{1}{8} u_{i-3}}{h^{3}}\right] .
\end{aligned}
$$

and write the whole system in matrix form:

$$
\frac{d}{d t}[A U]=L(U)+B U
$$

where $L(u)$ is the high order spatial discrete formulation of $-f_{x}(u)$ obtained from WENO reconstruction. Finally, we obtain the matrix form of the semi-discrete system (1.3):

$$
\frac{d U}{d t}=A^{-1} L(U)+A^{-1} B U
$$

where

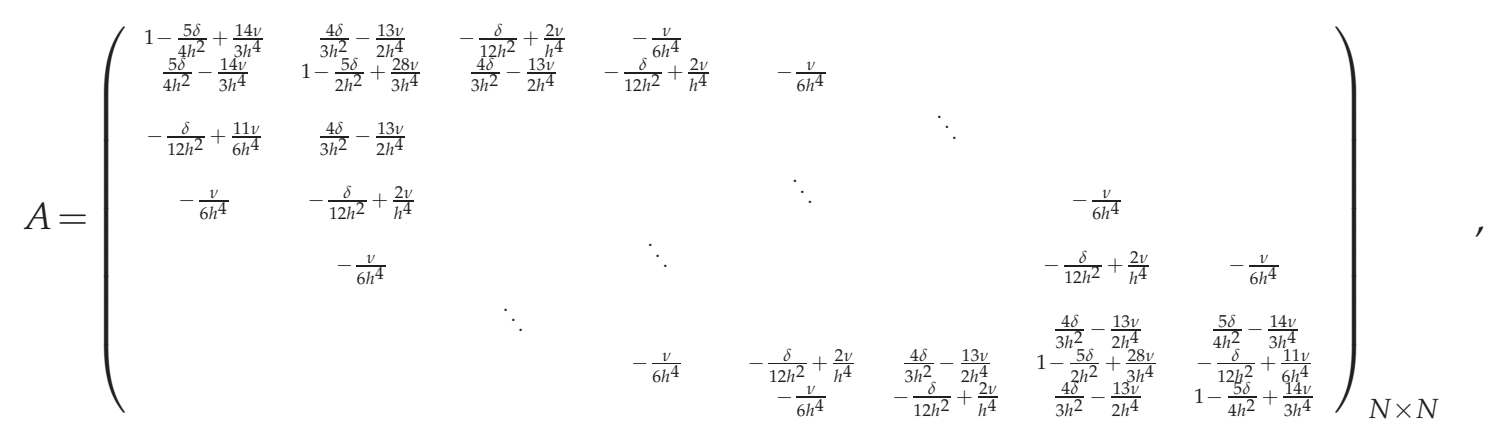


and

$$
B=\left(\begin{array}{cccccccc}
-\frac{13 \theta}{8 h^{3}}+\frac{\theta}{h^{3}}-\frac{\theta}{8 h^{3}} & \frac{13 \theta}{8 h^{3}} & -\frac{\theta}{h^{3}} & \frac{\theta}{8 h^{3}} & & & & \\
-\frac{13 \theta}{8 h^{3}}+\frac{\theta}{h^{3}}-\frac{\theta}{8 h^{3}} & 0 & \frac{13 \theta}{8 h^{3}} & -\frac{\theta}{h^{3}} & \frac{\theta}{8 h^{3}} & & & \\
\frac{\theta}{h^{3}}-\frac{\theta}{8 h^{3}} & -\frac{13 \theta}{8 h^{3}} & & & & \ddots & & \\
-\frac{\theta}{8 h^{3}} & \frac{\theta}{h^{3}} & & & \ddots & & \frac{\theta}{8 h^{3}} & \\
& -\frac{\theta}{8 h^{3}} & & \ddots & & & -\frac{\theta}{h^{3}} & \frac{\theta}{8 h^{3}} \\
& & \ddots & & & & \frac{13 \theta}{8 h^{3}} & -\frac{\theta}{h^{3}}+\frac{\theta}{8 h^{3}} \\
& & & -\frac{\theta}{8 h^{3}} & \frac{\theta}{h^{3}} & -\frac{13 \theta}{8 h^{3}} & 0 & \frac{13 \theta}{8 h^{3}}-\frac{\theta}{h^{3}}+\frac{\theta}{8 h^{3}} \\
& & & & -\frac{\theta}{8 h^{3}} & \frac{\theta}{h^{3}} & -\frac{13 \theta}{8 h^{3}} & \frac{13 \theta}{8 h^{3}}-\frac{\theta}{h^{3}}+\frac{\theta}{8 h^{3}}
\end{array}\right)_{N \times N}
$$

Since the wave amplitude near the boundaries tends to be constant for long wave behavior, so we use inflow and outflow boundary at n-th time level. In order to make sure the scheme is also third order at the boundary, we need three cell information outside of the computational domain for each side and add these ghost cell values into the first and last column of matrix B for numerical simplicity.

Further, we will use S-stage SSP IMEX-RK scheme for the temporal discretization, specifically S-stage SSP Explicit Runge-Kutta method for advection terms which are computed by third-order WENO reconstruction, and L-stable diagonally implicit RungeKutta (DIRK) method for stiff parts caused by high order spatial derivation. This method has the following form when it is applied to (3.5):

$$
\begin{aligned}
& U^{(m)}=U^{n}+\tau \sum_{q=1}^{m-1} \tilde{a}_{m q} A^{-1} L\left(U^{(q)}\right)+\tau \sum_{q=1}^{S} \hat{a}_{m q} A^{-1} B U^{(q)}, \\
& U^{n+1}=U^{n}+\tau \sum_{q=1}^{S} \tilde{b}_{q} A^{-1} L\left(U^{(q)}\right)+\tau \sum_{q=1}^{S} \hat{b}_{q} A^{-1} B U^{(q)} .
\end{aligned}
$$

with the double Butchar tableau

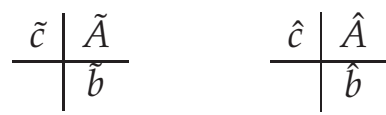

$$
\begin{aligned}
& \text { Explicit Implicit }
\end{aligned}
$$

where $\tilde{b}=\left(\tilde{b}_{1}, \tilde{b}_{2}, \cdots, \tilde{b}_{S}\right)^{T}, \tilde{c}=\left(\tilde{c}_{1}, \tilde{c}_{2}, \cdots, \tilde{c}_{S}\right)^{T}, \hat{b}=\left(\hat{b}_{1}, \hat{b}_{2}, \cdots, \hat{b}_{S}\right)^{T}, \hat{c}=\left(\hat{c}_{1}, \hat{c}_{2}, \cdots, \hat{c}_{S}\right)^{T}$ are coefficient vectors and $\tilde{A}=\left(\tilde{a}_{m q}\right), \tilde{a}_{m q}=0$ for $q \geq m$ and $\hat{A}=\left(\hat{a}_{m q}\right)$ are $S \times S$ matrices.

By using the above procedure, we present the algorithm for numerical solution.

- Explicit term

$$
U_{*}^{(m)}=U^{n}+\tau \sum_{q=1}^{m-2} \tilde{a}_{m q} A^{-1} L\left(U^{(q)}\right)+\tau \tilde{a}_{m, m-1} A^{-1} L\left(U^{(m-1)}\right),
$$


- Implicit term

$$
U^{(m)}=U_{*}^{(m)}+\tau \sum_{q=1}^{m-1} \hat{a}_{m q} A^{-1} B U^{(q)}+\tau \hat{a}_{m m} A^{-1} B U^{(m)},
$$

- Final solution at next time level

$$
U^{n+1}=U^{n}+\tau \sum_{q=1}^{S} \tilde{b}_{q} A^{-1} L\left(U^{(q)}\right)+\tau \sum_{q=1}^{S} \hat{b}_{q} A^{-1} B U^{(q)} .
$$

In this paper, we apply the third-order L-stable SSP IMEX Runge-Kutta method with the Butchar tableau given as in [20]:

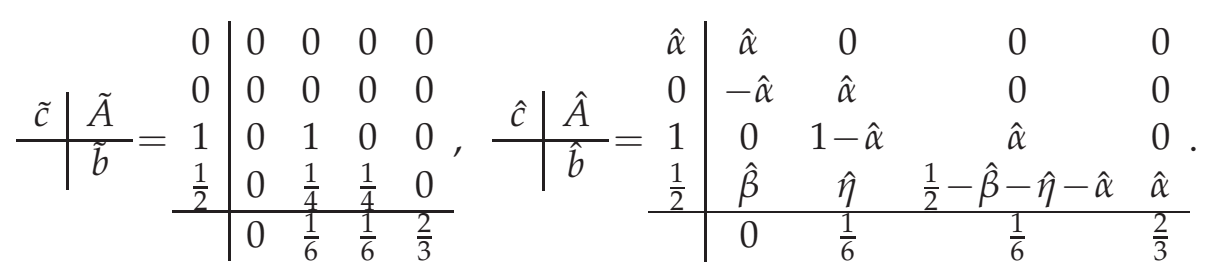

with $\hat{\alpha}=0.24169426078821, \hat{\beta}=\frac{\hat{\alpha}}{4}, \hat{\eta}=0.12915286960590$. Hence we have the following temporal operation:

$$
\begin{array}{ll}
m=1 & U_{*}^{(1)}=U^{n}, \\
& U^{(1)}=U_{*}^{(1)}+\tau \hat{a}_{11} A^{-1} B U^{(1)} . \\
m=2 & U_{*}^{(2)}=U^{n}+\tau \tilde{a}_{21} A^{-1} L\left(U^{(1)}\right) \\
& U^{(2)}=U_{*}^{(2)}+\tau \hat{a}_{21} A^{-1} B U^{(1)}+\tau \hat{a}_{22} A^{-1} B U^{(2)} . \\
m=3 & U_{*}^{(3)}=U^{n}+\tau \tilde{a}_{31} A^{-1} L\left(U^{(1)}\right)+\tau \tilde{a}_{32} A^{-1} L\left(U^{(2)}\right), \\
& U^{(3)}=U_{*}^{(3)}+\tau \hat{a}_{31} A^{-1} B U^{(1)}+\tau \hat{a}_{32} A^{-1} B U^{(2)}+\tau \hat{a}_{33} A^{-1} B U^{(3)} . \\
m=4 & U_{*}^{(4)}=U^{n}+\tau \tilde{a}_{41} A^{-1} L\left(U^{(1)}\right)+\tau \tilde{a}_{42} A^{-1} L\left(U^{(2)}\right)+\tau \tilde{a}_{43} A^{-1} L\left(U^{(3)}\right), \\
& U^{(4)}=U_{*}^{(4)}+\tau \hat{a}_{41} A^{-1} B U^{(1)}+\tau \hat{a}_{42} A^{-1} B U^{(2)}+\tau \hat{a}_{43} A^{-1} B U^{(3)}+\tau \hat{a}_{44} A^{-1} B U^{(4)} . \\
U^{n+1}= & U^{n}+\tau \tilde{b}_{1} A^{-1} L\left(U^{(1)}\right)+\tau \tilde{b}_{2} A^{-1} L\left(U^{(2)}\right)+\tau \tilde{b}_{3} A^{-1} L\left(U^{(3)}\right)+\tau \tilde{b}_{4} A^{-1} L\left(U^{(4)}\right) \\
& +\tau \hat{b}_{1} A^{-1} B U^{(1)}+\tau \hat{b}_{2} A^{-1} B U^{(2)}+\tau \hat{b}_{3} A^{-1} B U^{(3)}+\tau \hat{b}_{4} A^{-1} B U^{(4)} .
\end{array}
$$

\section{Numerical results}

In this section, we will discuss computational results of the scheme (3.9) on some numerical examples for the solitary wave solution and shock wave solution of Rosenau$\mathrm{KdV}$ equation and Rosenau-KdV-RLW equation. 
Table 1: Errors and rates of convergence when $C F L=1, h=\tau$ at $T=20$ for Example 4.1.

\begin{tabular}{||c|cccc||}
\hline$h$ & $L_{1}$ & order & $L_{\infty}$ & order \\
\hline 0.2 & $3.2085 \mathrm{e}-05$ & & $4.7295 \mathrm{e}-04$ & \\
0.1 & $3.7105 \mathrm{e}-06$ & 3.1122 & $5.4363 \mathrm{e}-05$ & 3.1210 \\
0.05 & $4.1275 \mathrm{e}-07$ & 3.1683 & $5.9524 \mathrm{e}-06$ & 3.1911 \\
0.025 & $4.2519 \mathrm{e}-08$ & 3.2791 & $6.0110 \mathrm{e}-07$ & 3.3078 \\
\hline
\end{tabular}

We consider the linear version of (1.3) with parameter $\delta=-1, v=1, \theta=1, \alpha+\varepsilon=1, p=1$. We obtain stability restriction $\frac{\Delta t}{\Delta x} \leq 1$ by standard Fourier analysis and computation, and take $C F L=1$ for all numerical simulation in this paper.

Example 4.1. Consider Rosenau-KdV equation (1.2) with parameters $\delta=0, v=1, \alpha=1, \theta=$ $1, \varepsilon=\frac{1}{2}, p=2$ :

$$
u_{t}+u_{x x x x t}+u_{x x x}+u_{x}+\left(\frac{1}{2} u^{2}\right)_{x}=0, \quad x \in[-70,100], t \in[0, T]
$$

and choose the initial condition to be $u(x, 0)=M \operatorname{sech}^{\frac{4}{p-1}}(W x)$, so that the analytical solitary wave solution of Rosenau-KdV equation is $u(x, t)=\operatorname{Msech}^{\frac{4}{p-1}}[W(x-V t)]$ as in [2] with wave width

$$
W=\frac{p-1}{p+1}\left[\frac{-\alpha v\left(p^{2}+2 p+5\right)+\sqrt{\alpha^{2} v^{2}\left(p^{2}+2 p+5\right)^{2}+16 \theta^{2} v(p+1)^{2}}}{32 \theta v}\right]^{\frac{1}{2}}
$$
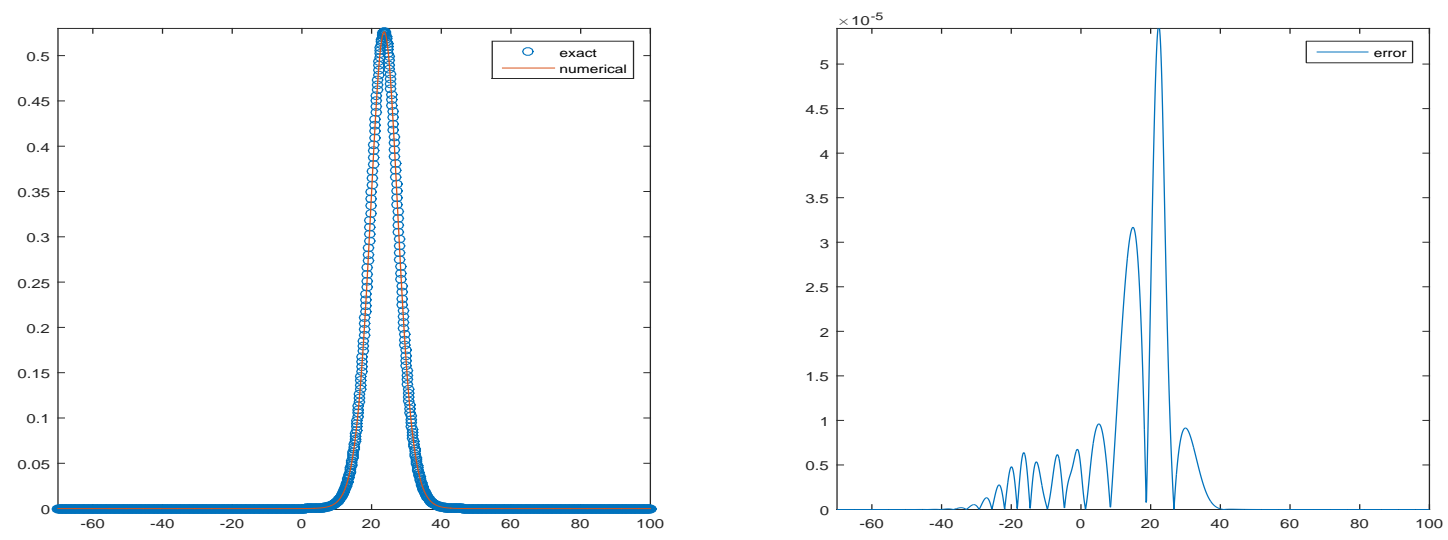

Figure 1: Wave graph of $u(x, t)$ at $T=20$ and numerical solution of Rosenau-KdV equation with $h=\tau=0.1$ at $T=20$ (left) and error (right) for Example 4.1. 


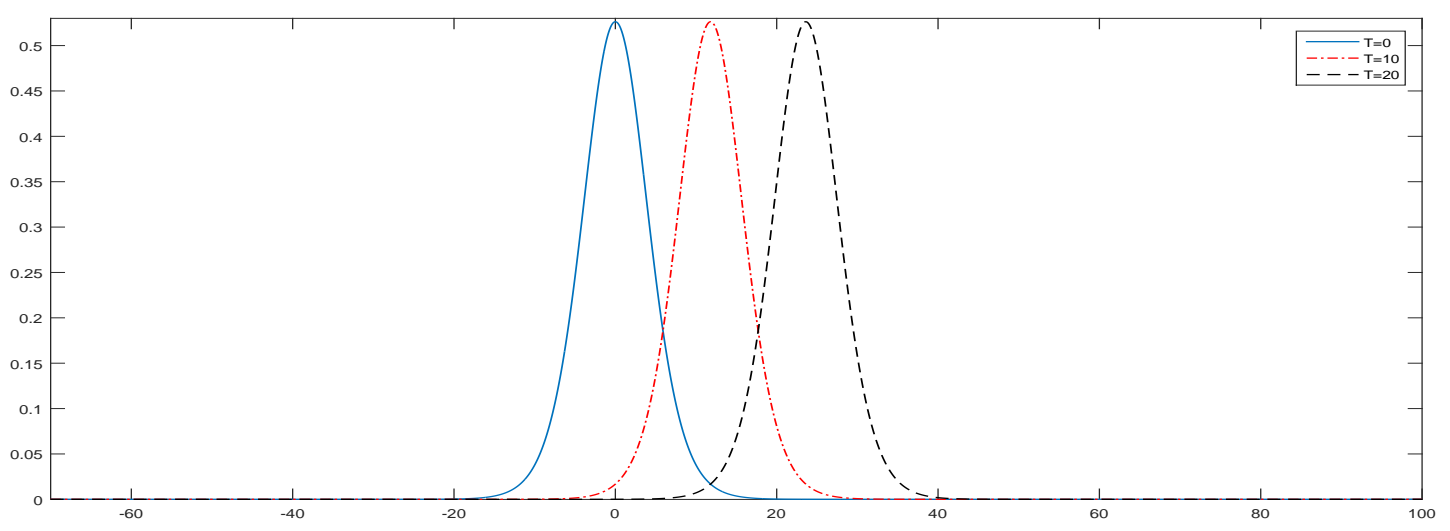

Figure 2: Numerical solution of Rosenau-KdV equation with $h=\tau=0.1$ at $T=0,10,20$ for Example 4.1. 1

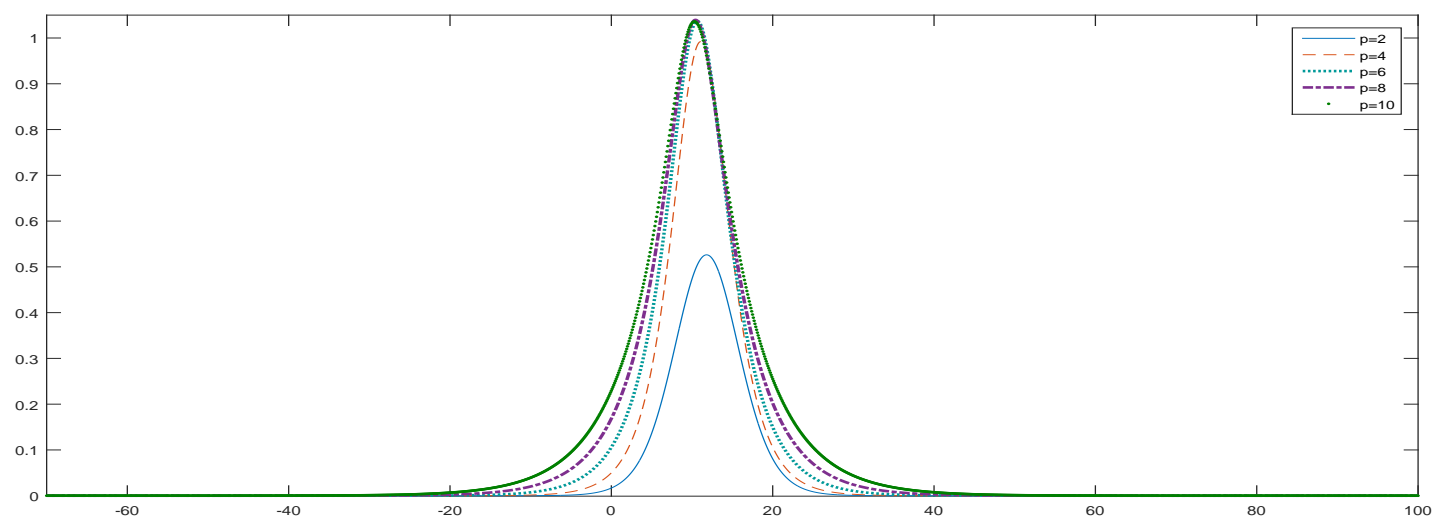

Figure 3: Numerical solution of Rosenau-KdV equation with $p=2,4,6,8,10, \epsilon=\frac{1}{p}$ and $h=\tau=0.1$ at $T=20$ for Example 4.1.

wave velocity

$$
V=\frac{\theta(p-1)^{2}}{4 v W^{2}\left(p^{2}+2 p+5\right)}
$$

and wave amplitude

$$
M=\left[\frac{\left[-\alpha v\left(p^{2}+2 p+5\right)+\sqrt{\alpha^{2} v^{2}\left(p^{2}+2 p+5\right)^{2}+16 \theta^{2} v(p+1)^{2}}\right](p+3)(3 p+1)}{16 v \epsilon(p+1)\left(p^{2}+2 p+5\right)}\right]^{\frac{1}{p-1}} .
$$

Errors and rates of convergence in terms of $L_{1}$ and $L_{\infty}$ at $T=20$ for $\tau=C F L \cdot h$ with $C F L=1$ in interval $x \in[-70,100]$ are listed in Table 1 for Example 4.1 . The third order accuracy of the numerical method is achieved as we expected in the theoretical procedure, 
Table 2: Comparison of $L_{\infty}$ errors at $T=20$ for Example 4.1.

\begin{tabular}{||c|cccc||}
\hline Scheme & $h=\tau=0.2$ & $h=\tau=0.1$ & $h=\tau=0.05$ & $h=\tau=0.025$ \\
\hline Scheme [12] & $7.8920 \mathrm{e}-04$ & $1.8771 \mathrm{e}-04$ & $4.6987 \mathrm{e}-05$ & $1.1751 \mathrm{e}-05$ \\
Scheme [11] $\left(-\frac{1}{3}\right)$ & $1.0192 \mathrm{e}-03$ & $2.5411 \mathrm{e}-04$ & $6.3501 \mathrm{e}-05$ & $1.5876 \mathrm{e}-05$ \\
Scheme [11] $\left(\frac{1}{3}\right)$ & $4.9510 \mathrm{e}-04$ & $1.2372 \mathrm{e}-04$ & $3.0934 \mathrm{e}-05$ & $7.7336 \mathrm{e}-05$ \\
Scheme $(3.9)$ & $4.7295 \mathrm{e}-04$ & $5.4363 \mathrm{e}-05$ & $5.9524 \mathrm{e}-06$ & $6.0110 \mathrm{e}-07$ \\
\hline
\end{tabular}

Table 3: $L_{1}, L_{\infty}$ errors of numerical solutions for Rosenau-KdV equation with $h=\tau, T=20, \varepsilon=\frac{1}{p}$ for Example 4.1.

\begin{tabular}{||c|ccc|ccc||}
\hline \multirow{2}{*}{$h$} & \multicolumn{3}{|c|}{$L_{1}$} & \multicolumn{3}{c||}{$L_{\infty}$} \\
\cline { 2 - 7 } & 0.2 & 0.1 & 0.05 & 0.2 & 0.1 & 0.05 \\
\hline 2 & $3.2085 \mathrm{e}-05$ & $3.7105 \mathrm{e}-06$ & $4.1275 \mathrm{e}-07$ & $4.7295 \mathrm{e}-04$ & $5.4363 \mathrm{e}-05$ & $5.9524 \mathrm{e}-06$ \\
& & 3.1122 & 3.1683 & & 3.1210 & 3.1911 \\
\hline 4 & $1.2393 \mathrm{e}-04$ & $1.5684 \mathrm{e}-05$ & $1.9626 \mathrm{e}-06$ & $1.7057 \mathrm{e}-03$ & $2.1598 \mathrm{e}-04$ & $2.6539 \mathrm{e}-05$ \\
& & 2.9822 & 2.9984 & & 2.9814 & 3.0247 \\
\hline 6 & $1.5405 \mathrm{e}-04$ & $1.9329 \mathrm{e}-05$ & $2.5037 \mathrm{e}-06$ & $2.0945 \mathrm{e}-03$ & $2.6151 \mathrm{e}-04$ & $3.4664 \mathrm{e}-05$ \\
& & 2.9946 & 2.9487 & & 3.0017 & 2.9154 \\
\hline 8 & $1.5433 \mathrm{e}-04$ & $1.9268 \mathrm{e}-05$ & $2.5311 \mathrm{e}-06$ & $2.1308 \mathrm{e}-03$ & $2.6494 \mathrm{e}-04$ & $3.5590 \mathrm{e}-05$ \\
& & 3.0017 & 2.9284 & & 3.0077 & 2.8961 \\
\hline 10 & $1.4411 \mathrm{e}-04$ & $1.8034 \mathrm{e}-05$ & $2.4683 \mathrm{e}-06$ & $2.0081 \mathrm{e}-03$ & $2.4933 \mathrm{e}-04$ & $3.3461 \mathrm{e}-05$ \\
& & 2.9983 & 2.8691 & & 3.0097 & 2.8975 \\
\hline
\end{tabular}

and works well with large time step. We can observe from the left of Figure 1 that the solitary wave curve matches excellently with exact solution when $h=\tau=0.1$ at $T=20$. From the right of Figure 1, it can be seen that error mostly generates at two sides of the solidary wave.

We compare the $L_{\infty}$ errors of our scheme with the results of other three numerical schemes [11,12] under various mesh steps $h=\tau$ at $T=20$ in Table 2. The better computational accuracy of the present scheme can be seen with the smallest error among other schemes referred above. The solitary wave graphs at $T=10,20$ agree with the one at $T=0$ quite well. The solitary wave curve propagates with constant speed $\mathrm{V}$ to the right through time $\mathrm{T}$ in Figure 2.

To observe the effect of power law nonlinear term to the solitary wave of Rosenau$\mathrm{KdV}$ equation, we draw the wave curves for $p=2,4,6,8,10$ and $\varepsilon=\frac{1}{p}$ with $h=\tau=0.1$ at $T=20$ in Figure 3, The wave amplitude and width are increasing while $p$ increases. We compute $L_{1}, L_{\infty}$ errors for $p=2,4,6,8,10$ and $\varepsilon=\frac{1}{p}$ at $T=20$ on three different meshes in Table 3 and also achieve third-order convergence in each case.

Next, we refer shock wave solutions of Rosenau-KdV equation from [3] which is available only for two particular values of power law nonlinearity parameter $p=3,5$. Our 
scheme simulates this wave phenomena efficiently with its essentially non-oscillatory property.

Example 4.2. Consider Rosenau-KdV equation (1.2) with parameters $\delta=0, v=-10, \alpha=$ $0.05, \theta=0.001, \epsilon=-5, p=5$ :

$$
u_{t}+0.05 u_{x}+0.001 u_{x x x}-10 u_{x x x x t}-5\left(u^{5}\right)_{x}=0, \quad x \in[-10,10], t \in[0, T] .
$$

and choose the initial condition to be $u_{0}(x)=M \tanh (W x)$, so that the analytical shock wave solution of Rosenau-KdV equation for $p=5$ is $u(x, t)=M \tanh [W(x-V t)]$ as in [3] with

$$
W=\frac{1}{2}\left[\frac{5 \alpha}{3 \theta}-\frac{1}{3 \theta} \sqrt{\frac{6 \theta^{2}+25 \alpha^{2} v}{v}}\right]^{\frac{1}{2}}, \quad V=\frac{\alpha-2 \theta W^{2}}{16 v W^{4}+1},
$$

and $M=W\left(\frac{24 V v}{\varepsilon}\right)^{\frac{1}{4}}$.

These parameters have to be chosen carefully to make sure that the three quantities are all real. In Table 4, we show errors and rates of convergence to highlight the efficiency of the WENO reconstruction for shock wave in the case of $p=5$. Figure 4 displays the

Table 4: Errors and rates of convergence when $C F L=1, h=\tau$ at $T=10$ for Example 4.2.

\begin{tabular}{||c|cccc||}
\hline$h$ & $L_{1}$ & order & $L_{\infty}$ & order \\
\hline 0.2 & $2.5912 \mathrm{e}-03$ & & $7.8227 \mathrm{e}-03$ & \\
0.1 & $7.2593 \mathrm{e}-04$ & 1.8357 & $2.1876 \mathrm{e}-03$ & 1.8383 \\
0.05 & $1.2977 \mathrm{e}-04$ & 2.4839 & $3.8145 \mathrm{e}-04$ & 2.5198 \\
0.025 & $1.6055 \mathrm{e}-05$ & 3.0148 & $4.6823 \mathrm{e}-05$ & 3.0262 \\
\hline
\end{tabular}
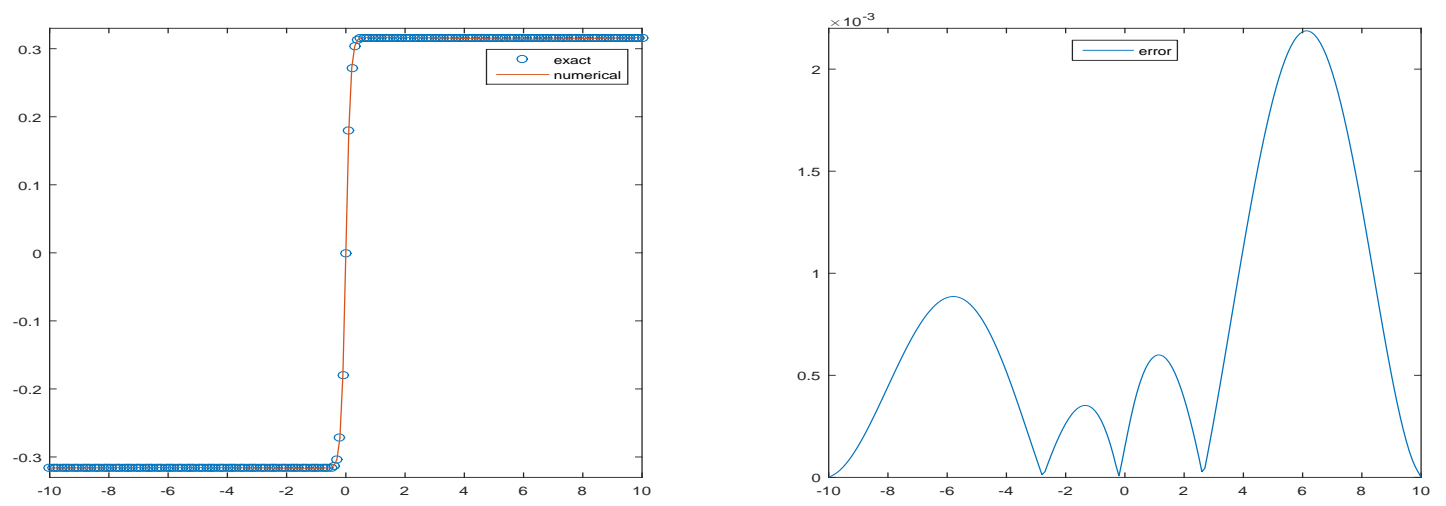

Figure 4: Wave graph of $u(x, t)$ at $T=10$ and numerical solution of Rosenau-KdV equation with $h=\tau=0.1, p=5$ at $T=10$ (left) and error (right) for Example 4.2. 
shock wave at $T=10$ with $h=\tau=0.1$ on the left and error on the right. As we can see there is no oscillatory nearby the stiff region.

Example 4.3. Consider Rosenau-KdV equation (1.2) with parameters $\delta=0, v=-10, \alpha=$ $0.4, \theta=0.01, \epsilon=-3, p=3$ :

$$
u_{t}+0.4 u_{x}+0.01 u_{x x x}-10 u_{x x x x t}-3\left(u^{3}\right)_{x}=0, \quad x \in[-10,10], t \in[0, T] .
$$

and choose the initial condition to be $u_{0}(x)=M \tanh ^{2}(W x)$, so that the analytical shock wave solution of Rosenau-KdV equation for $p=3$ is $u(x, t)=M \tanh ^{2}[W(x-V t)]$ as in [3] with

$$
W=\frac{1}{2}\left[\frac{10 \alpha}{23 \theta}-\frac{1}{23 \theta} \sqrt{\frac{100 \alpha^{2} v+46 \theta^{2}}{v}}\right]^{\frac{1}{2}}, \quad V=\frac{\alpha-8 \theta W^{2}}{136 v W^{4}+1},
$$

and $M=2 W^{2}\left(\frac{30 V v}{\varepsilon}\right)^{\frac{1}{2}}$.

Table 5: Errors and rates of convergence when $C F L=1, h=\tau$ at $T=10$ for Example 4.3.

\begin{tabular}{||c|cccc||}
\hline $\mathrm{h}$ & $L_{1}$ & order & $L_{\infty}$ & order \\
\hline 0.2 & $2.1529 \mathrm{e}-03$ & & $5.7931 \mathrm{e}-03$ & \\
0.1 & $7.5346 \mathrm{e}-04$ & 1.5147 & $2.2966 \mathrm{e}-03$ & 1.3348 \\
0.05 & $1.3853 \mathrm{e}-04$ & 2.4433 & $4.5664 \mathrm{e}-04$ & 2.3304 \\
0.025 & $1.7016 \mathrm{e}-05$ & 3.0252 & $5.9426 \mathrm{e}-05$ & 2.9419 \\
\hline
\end{tabular}

In Table 5, we give error and rate of convergence for shock wave when $p=3$. Obviously here we achieve order that smaller than three at first, but it will converge to three
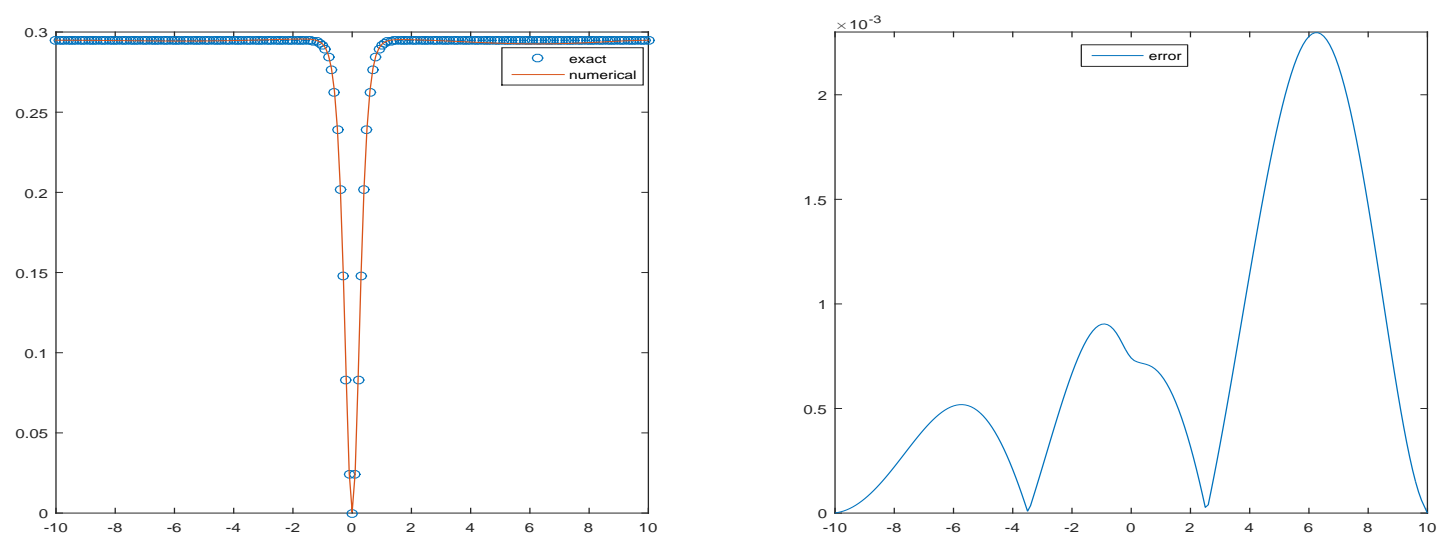

Figure 5: Wave graph of $u(x, t)$ at $T=10$ and numerical solution of Rosenau-KdV equation with $h=\tau=0.1, p=3$ at $T=10$ (left) and error (right) for Example 4.3. 
Table 6: The Comparison of $L_{\infty}$ errors with $C F L=1, h=\tau$ at $T=10$ between four different schemes for Example 4.4

\begin{tabular}{||c|cccc||}
\hline$(h, \tau)$ & $(0.4,0.4)$ & $(0.2,0.2)$ & $(0.1,0.1)$ & $(0.05,0.05)$ \\
\hline Scheme [11] $\left(-\frac{1}{3}\right)$ & $6.3995 \mathrm{e}-02$ & $1.5250 \mathrm{e}-02$ & $3.7908 \mathrm{e}-03$ & $9.4866 \mathrm{e}-04$ \\
Scheme [11] $\left(\frac{1}{3}\right)$ & $1.2031 \mathrm{e}-01$ & $3.0396 \mathrm{e}-02$ & $7.6198 \mathrm{e}-03$ & $1.9070 \mathrm{e}-03$ \\
Scheme [12] & $1.1194 \mathrm{e}-01$ & $2.8840 \mathrm{e}-02$ & $7.2669 \mathrm{e}-03$ & $1.8199 \mathrm{e}-03$ \\
Scheme(3.9) & $5.4870 \mathrm{e}-02$ & $7.6075 \mathrm{e}-03$ & $9.8854 \mathrm{e}-04$ & $1.2479 \mathrm{e}-04$ \\
\hline
\end{tabular}

Table 7: Errors and rates of convergence with $C F L=1, h=\tau$ at $T=10$ for Example 4.4.

\begin{tabular}{||c|cccc||}
\hline$h$ & $L_{1}$ & rate & $L_{\infty}$ & rate \\
\hline 0.2 & $8.4789 \mathrm{e}-04$ & & $7.6075 \mathrm{e}-03$ & \\
0.1 & $1.1027 \mathrm{e}-04$ & 2.9428 & $9.8854 \mathrm{e}-04$ & 2.9441 \\
0.05 & $1.3908 \mathrm{e}-05$ & 2.9871 & $1.2479 \mathrm{e}-04$ & 2.9858 \\
0.025 & $1.7632 \mathrm{e}-06$ & 2.9796 & $1.5496 \mathrm{e}-05$ & 3.0096 \\
\hline
\end{tabular}

eventually as the mesh is refined. Figure 5 displays the shock wave at $T=10$ when $h=\tau=0.1$ on the left and error on the right.

Example 4.4. Consider Rosenau-KdV-RLW equation (1.3) with parameters $\delta=-1, v=$ $1, \alpha=1, \theta=1, \varepsilon=\frac{1}{2}, p=2$ :

$$
u_{t}-u_{x x t}+u_{x x x x t}+u_{x}+u_{x x x}+\frac{1}{2}\left(u^{2}\right)_{x}=0, \quad x \in[-40,60], t \in[0, T] .
$$

and choose the initial condition to be $u(x, 0)=\operatorname{Msech}^{\frac{4}{p-1}}(W x)$, so that the analytical solitary wave solution of Rosenau-KdV-RLW equation is $u(x, t)=\operatorname{Msech}^{\frac{4}{p-1}}[W(x-V t)]$ as in [9] with

$$
D=\sqrt{\alpha^{2} v^{2}\left(p^{2}+2 p+5\right)^{2}+16(p+1)^{2} \theta v(\theta-\alpha \delta)}
$$

wave width

$$
W=\frac{p-1}{p+1} \sqrt{\frac{D-\left(p^{2}+2 p+5\right) \alpha v}{32 \theta v}}
$$

wave speed

$$
V=\frac{\theta(p-1)^{2}}{(p-1)^{2} \delta+4 v W^{2}\left(p^{2}+2 p+5\right)}
$$

and amplitude

$$
M=\left[\frac{8(p+1)(p+3)(3 p+1) \theta v W^{4}}{\varepsilon(p-1)^{2}\left((p-1)^{2} \delta+4\left(p^{2}+2 p+5\right) v W^{2}\right)}\right]^{\frac{1}{p-1}} .
$$



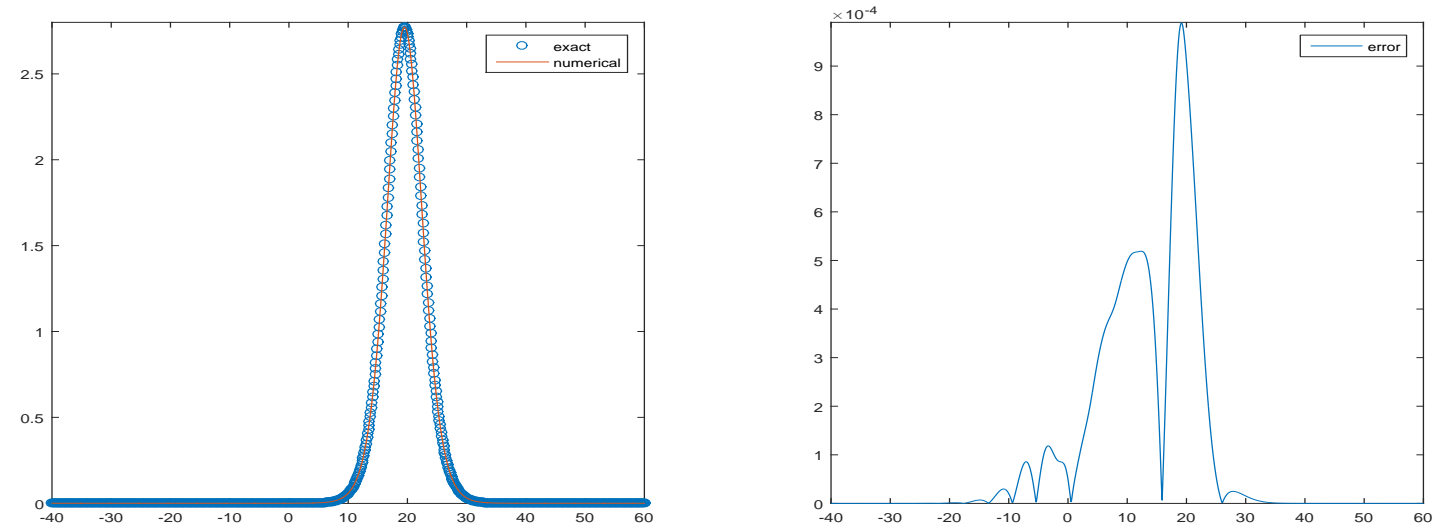

Figure 6: Wave graph of $u(x, t)$ at $T=10$ and numerical solution of Rosenau-KdV-RLW equation with $h=\tau=0.1$ at $T=10$ (left) and error (right) for Example 4.4.

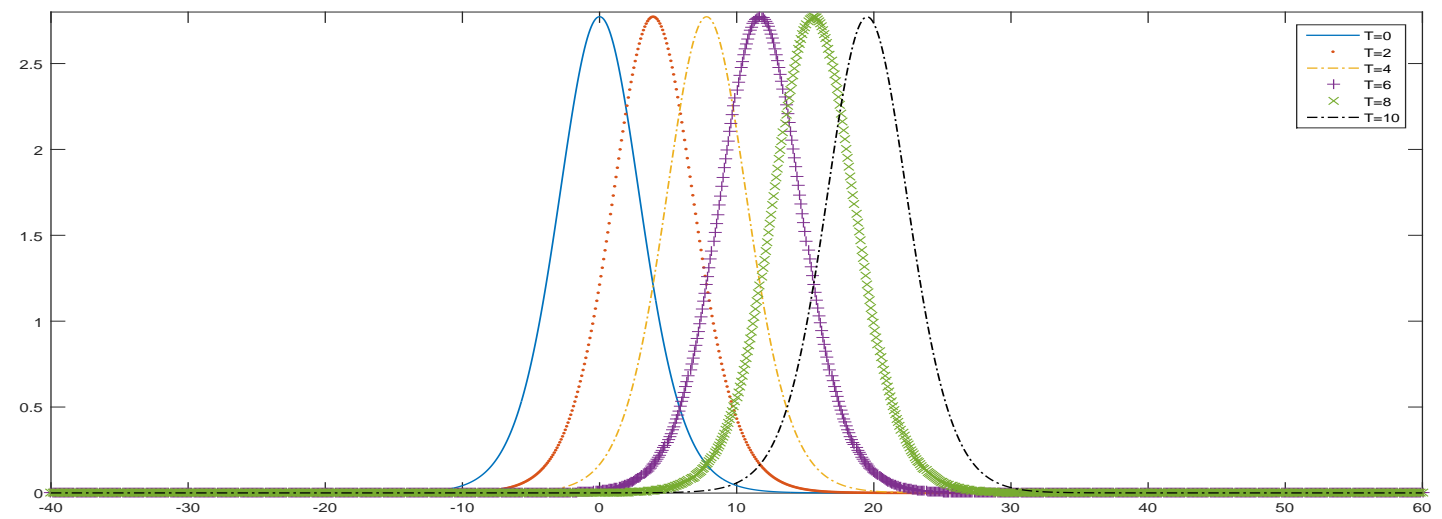

Figure 7: Numerical solution of Rosenau-KdV-RLW equation with $h=\tau=0.1$ at $T=2,4,6,8,10$ for Example 4.4.

The $L_{\infty}$ errors of the numerical solutions at $T=10$ under various mesh steps $h=\tau$ are listed in Table 6 and compare with other three types of schemes studied earlier about the same equation, which shows that our scheme has the smallest error in any cases.

On the left of Figure 6, the numerical wave curve totally matches with the analytical solidary solution at $T=10$ with mesh $h=\tau=0.1$ over the interval $x \in[-40,60]$ and the corresponding distribution of the error is drawn for solitary wave in the right of Figure 6.

As shown in Table 7, the third-order convergence of the numerical solutions is verified at $T=10$ for the solitary wave problem of the Rosenau-KdV-RLW equation. In Figure 7, perspective views of the traveling solutions are graphed at various time levels for $h=\tau=$ 0.1 .

In order to observe the effect of power law nonlinear term to the solidary wave of Rosenau-KdV-RLW equation, The $L_{1}, L_{\infty}$ errors and third-order convergence for $p=$ 


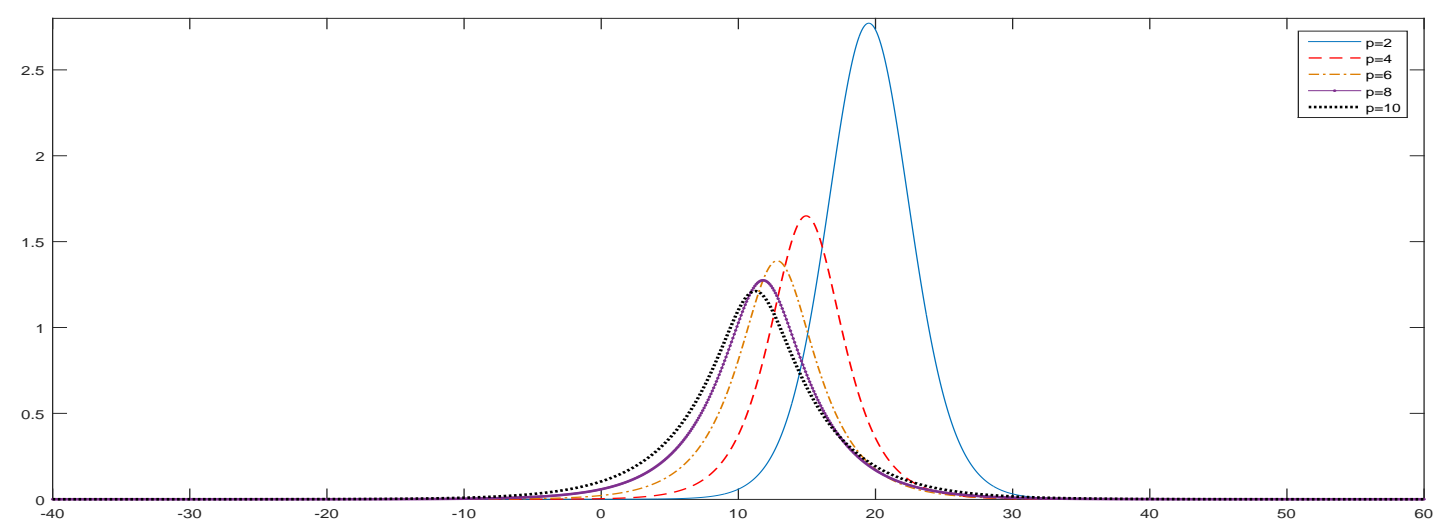

Figure 8: Numerical solution of Rosenau-KdV-RLW equation with $p=2,4,6,8,10, \epsilon=\frac{1}{p}$ and $h=\tau=0.1$ at $T=10$ for Example 4.4.

Table 8: $L_{1}, L_{\infty}$ errors of numerical solutions for Rosenau-KdV-RLW equation with $h=\tau, T=10, \varepsilon=\frac{1}{p}$ for Example 4.4 .

\begin{tabular}{|c|c|c|c|c|c|c|}
\hline & \multicolumn{3}{|c|}{$L_{1}$} & \multicolumn{3}{|c|}{$L_{\infty}$} \\
\hline & 0.2 & 0.1 & 0.05 & 0.2 & 0.1 & 0.05 \\
\hline 2 & $8.4789 \mathrm{e}-04$ & $\begin{array}{c}1.1027 \mathrm{e}-04 \\
2.9428\end{array}$ & $\begin{array}{c}1.3908 \mathrm{e}-05 \\
2.9871\end{array}$ & $7.6075 e-03$ & $\begin{array}{c}9.8854 \mathrm{e}-04 \\
2.9440\end{array}$ & $\begin{array}{c}1.2479 \mathrm{e}-04 \\
2.9858\end{array}$ \\
\hline 4 & $1.9237 \mathrm{e}-03$ & $\begin{array}{c}2.5823 \mathrm{e}-04 \\
2.8971\end{array}$ & $\begin{array}{c}3.2477 \mathrm{e}-05 \\
2.9912\end{array}$ & $2.1641 \mathrm{e}-02$ & $\begin{array}{c}2.8915 \mathrm{e}-03 \\
2.9039\end{array}$ & $\begin{array}{c}3.6262 \mathrm{e}-04 \\
2.9953\end{array}$ \\
\hline 6 & $1.9993 \mathrm{e}-03$ & $\begin{array}{c}2.7505 \mathrm{e}-04 \\
2.8617\end{array}$ & $\begin{array}{c}3.4557 \mathrm{e}-05 \\
2.9926\end{array}$ & $2.3003 e-02$ & $\begin{array}{c}3.1681 \mathrm{e}-03 \\
2.8601\end{array}$ & $\begin{array}{c}3.9806 \mathrm{e}-04 \\
2.9925\end{array}$ \\
\hline 8 & $1.8389 \mathrm{e}-03$ & $\begin{array}{c}2.5682 \mathrm{e}-04 \\
2.8400\end{array}$ & $\begin{array}{c}3.2327 \mathrm{e}-05 \\
2.9899\end{array}$ & $2.0847 \mathrm{e}-02$ & $\begin{array}{c}2.9226 \mathrm{e}-03 \\
2.8345\end{array}$ & $\begin{array}{c}3.6689 \mathrm{e}-04 \\
2.9938\end{array}$ \\
\hline 10 & $1.6504 \mathrm{e}-03$ & $\begin{array}{c}2.3332 \mathrm{e}-04 \\
2.8224\end{array}$ & $\begin{array}{c}3.0234 \mathrm{e}-05 \\
2.9481\end{array}$ & $1.8439 \mathrm{e}-02$ & $\begin{array}{c}2.6109 \mathrm{e}-03 \\
2.8202\end{array}$ & $\begin{array}{c}3.2730 \mathrm{e}-04 \\
2.9958\end{array}$ \\
\hline
\end{tabular}

$2,4,6,8,10$ and $\varepsilon=1 / p$ on three different mesh are listed in Table 8 . We draw the wave curves for these $p$ at $T=10$ with $C F L=1, h=\tau=0.1$ and $\varepsilon=1 / p$ in the interval $x \in[-40,60]$ for give further description in Figure 8. It can be observed that wave amplitude and speed decreases along with $p$ increases, this also fits the power law.

Based on earlier studies on the shock solution of the Rosenau-KdV equation, the shock wave solutions for the Rosenau-KdV-RLW equation were extracted by balancing principle only for $p=3$ and $p=5$ in [9]. Here we will review related formulation for wave amplitude, width, velocity mentioned in $[9,10]$, and then simulate both cases numerically 
as example.

Example 4.5. Consider Rosenau-KdV-RLW equation (1.3) with parameters $\delta=1, v=-0.001$, $\alpha=0.01, \theta=0.001, \varepsilon=-1, p=3$ :

$$
u_{t}+0.01 u_{x}+0.001 u_{x x x}+u_{x x t}-0.001 u_{x x x x t}-\left(u^{3}\right)_{x}=0, \quad x \in[-10,10], t \in[0, T] .
$$

and choose the initial condition to be $u_{0}(x)=M \tanh ^{2}(W x)$, so that the analytical shock wave solution of Rosenau-KdV-RLW equation for $p=3$ is $u(x, t)=M \tanh ^{2}[W(x-V t)]$ as in [9] with

$$
W=\left[\frac{10 \alpha v-\sqrt{100 \alpha^{2} v^{2}+46 \theta v(\theta-\alpha \delta)}}{92 \theta v}\right]^{\frac{1}{2}}, \quad V=\frac{\alpha-8 \theta W^{2}}{136 v W^{4}-8 \delta W^{2}+1},
$$

and $M=2 W^{2}\left(\frac{30 V v}{\varepsilon}\right)^{\frac{1}{2}}$.

In Table 9, the error comparisons in $L_{\infty}, L_{1}$ are obtained by present method for shock wave solution in the case of $p=3$ of the Rosenau-KdV-RLW equation in interval $x \in$ $[-10,10]$ with $h=\tau=0.2,0.1,0.05,0.025$ respectively and the simulations are run up to

Table 9: Errors and rates of convergence with $C F L=1, h=\tau$ at $T=10$ for Example 4.5.

\begin{tabular}{||c|cccc||}
\hline$h$ & $L_{1}$ & rate & $L_{\infty}$ & rate \\
\hline 0.2 & $8.0600 \mathrm{e}-06$ & & $1.6909 \mathrm{e}-04$ & \\
0.1 & $7.8356 \mathrm{e}-07$ & 3.3627 & $2.8062 \mathrm{e}-05$ & 2.5911 \\
0.05 & $6.7878 \mathrm{e}-08$ & 3.5290 & $2.5392 \mathrm{e}-06$ & 3.4662 \\
0.025 & $6.9675 \mathrm{e}-09$ & 3.2842 & $2.3362 \mathrm{e}-07$ & 3.4421 \\
\hline
\end{tabular}
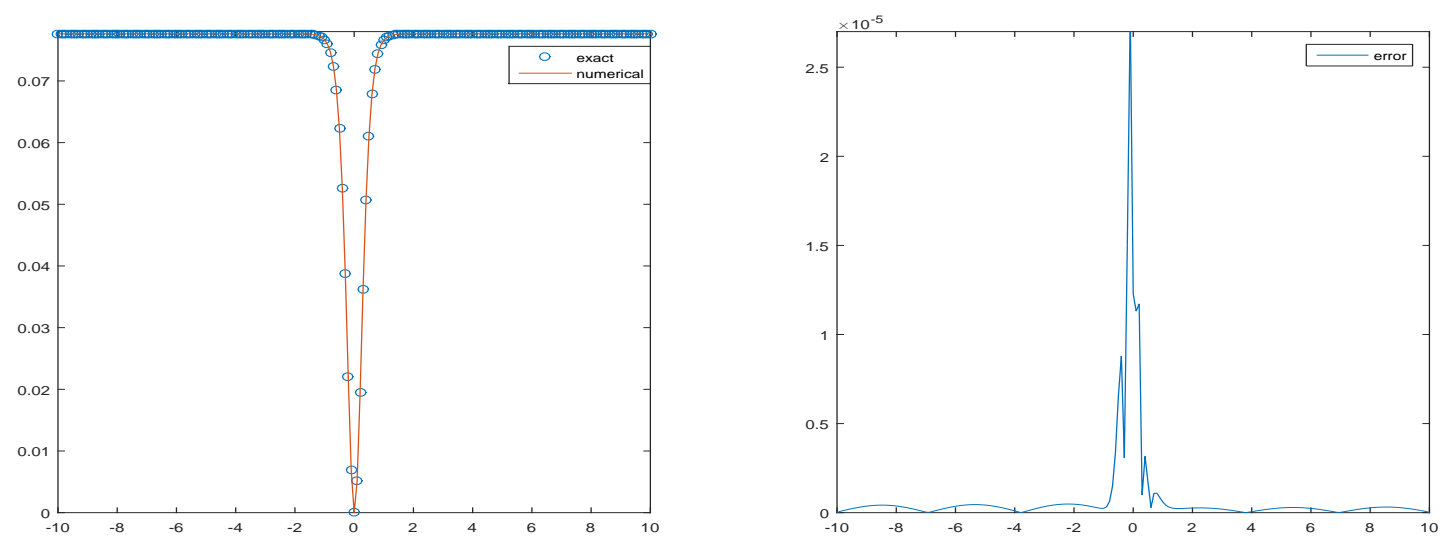

Figure 9: Wave graph of $u(x, t)$ at $T=10$ and numerical solution of Rosenau-KdV-RLW equation with $h=\tau=$ $0.1, p=3$ at $T=10$ (left) and error (right) for Example 4.5. 
time $T=10$ to obtain the error norms. It can be easily found that the errors are small, and the third-order convergence of the numerical solutions are also verified. From Figure 9, we can catch the point that numerical solution fits with exact one, and numerical method approximate the exact solution even in stiff concave region successfully.

Example 4.6. Consider Rosenau-KdV-RLW equation (1.3) with parameters $\delta=1, v=-10, \alpha=$ $0.05, \theta=0.001, \varepsilon=-5, p=5$ :

$$
u_{t}+u_{x x t}-10 u_{x x x x t}+0.05 u_{x}+0.001 u_{x x x}-5\left(u^{5}\right)_{x}=0 \quad x \in[-10,10], t \in[0, T] .
$$

and choose the initial condition to be $u_{0}(x)=M \tanh (W x)$ so that the analytical shock wave solution of Rosenau-KdV-RLW equation for $p=5$ is $u(x, t)=M \tanh [W(x-V t)]$ as in [9] with

$$
W=\left[\frac{5 \alpha v-\sqrt{25 \alpha^{2} v^{2}+6 \theta v(\theta-\alpha \delta)}}{12 \theta v}\right]^{\frac{1}{2}}, \quad V=\frac{\alpha-2 \theta W^{2}}{16 v W^{4}-2 \delta W^{2}+1},
$$

and $M=W\left(\frac{24 V v}{\varepsilon}\right)^{\frac{1}{4}}$.

The computation of error and order is completed at time $t=10$ when $C F L=1, h=\tau$ on various mesh in interval $x \in[-10,10]$ and displayed in Table 10. The numerical shock wave curve of Rosenau-KdV-RLW equation for $p=5$ is agree with exact solution with no oscillatory near the point $x=0$ when $h=\tau=0.1$ at $T=10$ on the left of Figure 10 .

Example 4.7. Consider Rosenau-KdV-RLW equation (1.3) with parameters $\delta=-0.01, v=$ $0.01, \alpha=0.01, \theta=0.01, \varepsilon=1, p=3$ :

$$
u_{t}-0.01 u_{x x t}+0.01 u_{x x x x t}+0.01 u_{x}+0.01 u_{x x x}+\left(u^{3}\right)_{x}=0 \quad x \in[0,120], t \in[0, T] .
$$
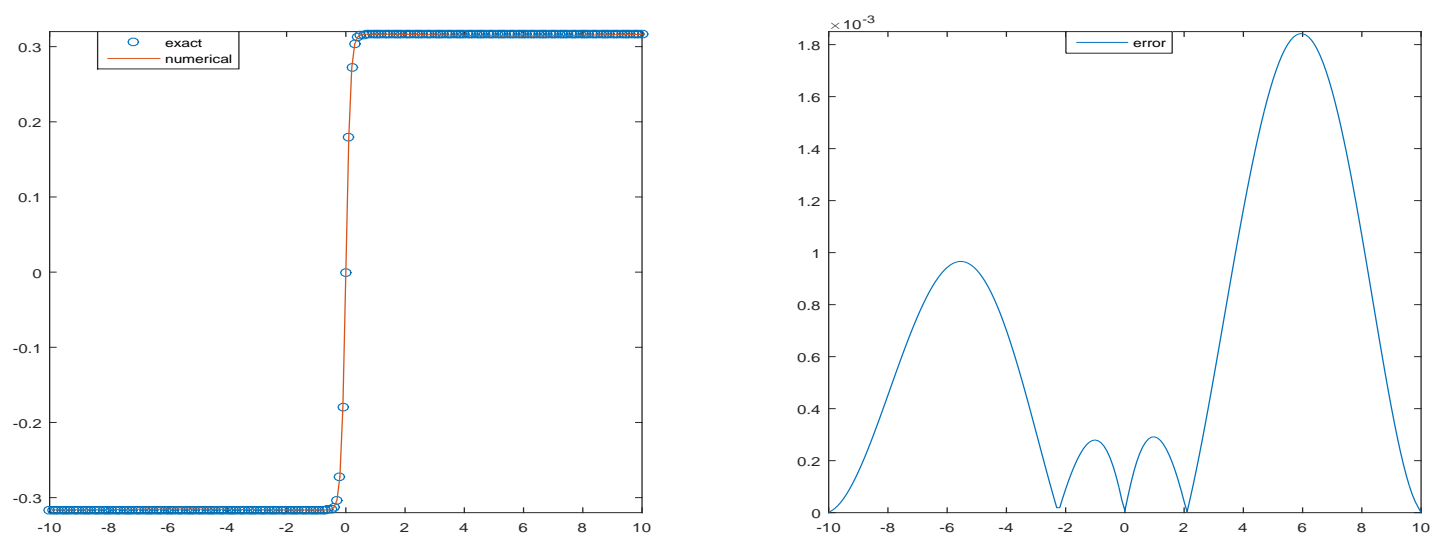

Figure 10: Wave graph of $u(x, t)$ at $T=10$ and numerical solution of Rosenau-KdV-RLW equation with $h=\tau=$ $0.1, p=5$ at $T=10$ (left) and error (right) for Example 4.6. 
Table 10: Errors and rates of convergence with $C F L=1, h=\tau$ at $T=10$ for Example 4.6.

\begin{tabular}{||c|cccc||}
\hline$h$ & $L_{1}$ & rate & $L_{\infty}$ & rate \\
\hline 0.2 & $2.1778 \mathrm{e}-03$ & & $5.2701 \mathrm{e}-03$ & \\
0.1 & $6.6553 \mathrm{e}-04$ & 1.7103 & $1.8419 \mathrm{e}-03$ & 1.5167 \\
0.05 & $1.2634 \mathrm{e}-04$ & 2.3972 & $3.6413 \mathrm{e}-04$ & 2.3387 \\
0.025 & $1.5887 \mathrm{e}-05$ & 2.9914 & $4.6009 \mathrm{e}-05$ & 2.9845 \\
\hline
\end{tabular}

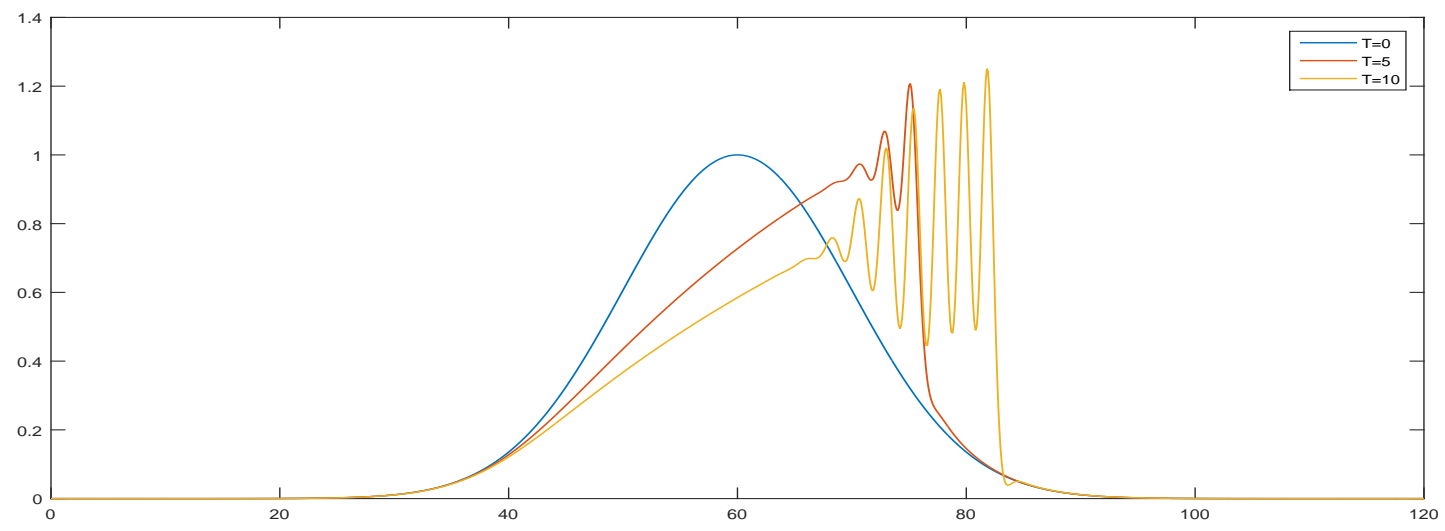

Figure 11: Wave graph of Rosenau-KdV-RLW equation at $T=0,5,10$ with the Maxwellian initial condition $u_{0}=\exp \left(-0.005(x-60)^{2}\right)$ for Example 4.7.

and Maxwellian initial condition to be $u_{0}(x)=\exp \left(-0.005(x-60)^{2}\right)$.

As a final example, we plot a high-frequency oscillatory behavior of $u(x, t)$ with above Maxwell initial condition in Figure 11 to illustrate the characteristic of dispersive shock wave behavior of (1.3). The steepening of the leading front repeats several times and decays until it is no longer present on the back of the wave.

\section{Concluding Remark}

To solve the solitary wave and shock wave problem of Rosenau-KdV equation and Rosenau-KdV-RLW equation, we use the third-order finite difference WENO reconstruction for advection terms, and central finite difference method for other terms in spatial discretization, then we use third-order SSP IMEX Runge-Kutta method for time discretization, in which the advection terms are treated by explicitly and remaining terms are treated by implicitly. In order to verify the effectiveness of the numerical scheme, some numerical examples are given for numerical experiment. Numerical simulations 
show that the method is very efficient with the advantages of non-oscillatory and looselyrestricted CFL condition.

\section{References}

[1] Rosenau P. Dynamics of dense discrete systems: high order effects. Progress Theor. Phys., 1988, 79(5): 1028-1042.

[2] Razborova P, Triki H, Biswas A. Perturbation of dispersive shallow water waves. Ocean Eng., 2013, 63(4): 1-7.

[3] Ebadi G, Mojaver A, Triki H, et al. Topological solitons and other solutions of the Rosenau-KdV equation with power law nonlinearity. Romanian J. Phy., 2013, 58(1-2): $1-10$.

[4] Atouani N, Omrani K. On the convergence of conservative difference schemes for the 2D generalized Rosenau-Korteweg de Vries equation. Appl. Math. Comput., 2015, 250: 832-847.

[5] Wang X, Dai W. A conservative fourth-order stable finite difference scheme for the generalized Rosenau-KdV equation in both 1D and 2D. J. Comput. Appl. Math., 2019, 355: 310-331.

[6] Wongsaijai B, Mouktonglang T, Sukantamala N, et al. Compact structure-preserving approach to solitary wave in shallow water modeled by the Rosenau-RLW equation. Appl. Math. Comput., 2019, 340: 84-100.

[7] Atouani N, Omrani K. Galerkin finite element method for the Rosenau-RLW equation. Comput. Math. Appl., 2013, 66(3): 289-303.

[8] Li S. Numerical analysis for fourth-order compact conservative difference scheme to solve the 3D Rosenau-RLW equation. Comput. Math. Appl., 2016, 72(9): 2388-2407.

[9] Razborova P, Ahmed B, Biswas A. Solitons, Shock waves and conservation laws of Rosenau-KdV-RLW equation with power law nonlinearity. Appl. Math. Inform. Sci., 2014, 8(2): 485-491.

[10] Razborova P, Kara A H, Biswas A. Additional conservation laws for Rosenau-KdV-RLW equation with power law nonlinearity by lie symmetry. Nonlinear Dyn., 2015, 79: 743748.

[11] Wongsaijai B, Poochinapan K. A three-level average implicit finite difference scheme to solve equation obtained by coupling the Rosenau-KdV equation and the Rosenau-RLW equation. Appl. Math. Comput., 2014, 245: 289-304.

[12] Wang X, Dai W. A three-level linear implicit conservative scheme for the Rosenau-KdVRLW equation. J. Comput. Appl. Math., 2017, 330: 295-306.

[13] Cai J, Liang H, Zhang C. Efficient high-order structure-preserving methods for the generalized Rosenau-type equation with power law nonlinearity. Commun. Nonlinear Sci. Numer. Simul., 2018, 59: 122-131.

[14] Apolinar-Fernndez A, Ramos J I. Numerical solution of the generalized, dissipative KdV-RLW-Rosenau equation with a compact method. Commun. Nonlinear Sci. Numer. Simul., 2018, 60: 165-183.

[15] Ascher U M, Ruuth S J, Spiteri R J. Implicit-explicit Runge-Kutta methods for timedependent partial differential equations. Appl. Numer. Math., 1997, 25(2/3): 151-167.

[16] Izzo G, Jackiewicz Z. Highly stable implicit-explicit Runge-Kutta methods. Appl. Numer. Math., 2017, 113: 71-92. 
[17] Boscarino S. On an accurate third order implicit-explicit Runge-Kutta method for stiff problems. Appl. Numer. Math., 2009, 59(7): 1515-1528.

[18] Pareschi L, Russo G. Implicit-explicit Runge-Kutta schemes for stiff systems of differential equations. Recent Trends in Numerical Analysis, 2000, 3: 269-289.

[19] Gottlieb S, Shu C-W, Tadmor E. Strong stability-preserving high-order time discretization methods. SIAM Review, 2001, 43: 89-112.

[20] Pareschi L, Russo G. Implicit-explicit Runge-Kutta schemes and applications to hyperbolic systems with relaxation. J. Sci. Comput., 2005, 25(1): 129-155.

[21] Shu C-W. Essentially non-oscillatory and weighted essentially non-oscillatory schemes for hyperbolic conservation laws. Advanced Numerical Approximation of Nonlinear Hyperbolic Equations, Springer, Berlin, Heidelberg, 1998: 325-432.

[22] Zhang Y-T, Shu C-W. ENO and WENO schemes. Handbook of Numerical Analysis, Elsevier, 2016, 17: 103-122.

[23] Vukovic S, Sopta L. ENO and WENO schemes with the exact conservation property for one-dimensional shallow water equations. Compu Physics, 2002, 179(2): 593-621. 